\title{
Some New Results on the Number of Paths
}

\author{
Beih S. El-Desouky1, Abdelfattah Mustafa ${ }^{1}$, E. M. Mahmoud ${ }^{2}$ \\ ${ }^{1}$ Mathematics Department, Faculty of Science, Mansoura University, Mansoura, Egypt \\ ${ }^{2}$ Mathematics Department, Faculty of Science, Aswan University, Aswan, Egypt \\ Email: b desouky@yahoo.com, abdelfatah mustafa@yahoo.com
}

Received 23 April 2015; accepted 1 June 2015; published 5 June 2015

Copyright (C) 2015 by authors and Scientific Research Publishing Inc.

This work is licensed under the Creative Commons Attribution International License (CC BY).

http://creativecommons.org/licenses/by/4.0/

(c) (i) Open Access

\section{Abstract}

Khidr and El-Desouky [1] derived a symmetric sum involving the Stirling numbers of the first kind through the process of counting the number of paths along a rectangular array $n \times m$ denoted by $A_{n m}$. We investigate the generating function for the general case and hence some special cases as well. The probability function of the number of paths along $A_{n m}$ is obtained. Moreover, the moment generating function of the random variable $X$ and hence the mean and variance are obtained. Finally, some applications are introduced.

\section{Keywords}

Stirling Numbers, Generating Function, Moment Generating Function, Comtet Numbers, Maple Program

\section{Introduction}

Let $\left\{a_{i}\right\}_{i=1}^{n}$ be a sequence of natural numbers $0 \leq a_{i} \leq n, i=1, \cdots, n$, and $A_{n m}$ be an $n \times m$ array associated with this sequence, whose entries $\alpha_{i j}=0,1$ such that

$$
\sum_{j=1}^{m} \alpha_{i j}=a_{i}, i=1,2, \cdots, n, j=1, \cdots, m .
$$

The path of order $k$ along $A_{n m}$ is defined to be a sequence of entries $\alpha_{1 j_{1}}, \cdots, \alpha_{n j_{n}}$ as follows

$$
\sum_{i=1}^{n} \alpha_{i j_{i}}=k, k=0,1, \cdots, n, j_{i}=1, \cdots, m, i=1, \cdots, n .
$$

The number of paths of order $k$ will be denoted by 


$$
g^{k}\left(a_{1}, \cdots, a_{n} ; m\right), k=0,1, \cdots n .
$$

By neglecting the last row in $A_{n m}$ and then reconsidering it, we get the recurrence

$$
g^{k}\left(a_{1}, \cdots, a_{n} ; m\right)=a_{n} g^{k-1}\left(a_{1}, \cdots, a_{n-1} ; m\right)+\left(m-a_{n}\right) g^{k}\left(a_{1}, \cdots, a_{n-1} ; m\right) .
$$

When $a_{i}=a, a$ is a constant, $i=1, \cdots, n$, then

$$
g^{k}(a, \cdots, a ; m) \cdots g^{k}(\bar{a}, m)=\left(\begin{array}{l}
n \\
k
\end{array}\right) a^{k}(m-a)^{n-k},
$$

and

$$
\sum_{k=0}^{n} g^{k}(\bar{a}, m)=m^{n}
$$

Khidr and El-Desouky [1] proved that, when $m=n$

$$
g^{k}\left(a_{1}, a_{2}, \cdots, a_{n} ; n\right)=(-1)^{k} \sum_{l=k}^{n}\left(\begin{array}{l}
l \\
k
\end{array}\right) s_{\bar{a}}(n, n-l) n^{n-l},
$$

where $s_{\bar{a}}(n, i)$ are the generalized Stirling numbers of the first kind associated with the sequence of real numbers $\bar{a}:=\left(a_{1}, a_{2}, \cdots, a_{n}\right)$, defined by [1]-[6],

$$
\left(x-a_{1}\right)\left(x-a_{2}\right) \cdots\left(x-a_{n}\right)=\sum_{i=0}^{n} s_{\bar{a}}(n, i) x^{i} .
$$

These numbers satisfy the recurrence relation

$$
s_{\bar{a}}(n, i)=s_{\bar{a}}(n-1, i-1)-a_{n} s_{\bar{a}}(n-1, i), s_{\bar{a}}(n, i)=0 \text { for } i>n .
$$

And

$$
\sum_{k=0}^{n} g^{k}(\bar{a}, n)=n^{n}
$$

Moreover, they introduced a special case of (3), when $a_{i}=i, i=1,2, \cdots, n$, then the number of paths of order $k, g^{k}(1, \cdots, n ; n)$ is denoted by $g_{n}^{k}(n)$; and proved that

$$
g_{n}^{k}(n)=(-1)^{k} \sum_{l=k}^{n}\left(\begin{array}{l}
l \\
k
\end{array}\right) s(n+1, n+1-l) n^{n-l},
$$

where $s(n, l)$ are the Stirling numbers of the first kind defined by, see [2] [3]

$$
x(x-1) \cdots(x-n+1)=\sum_{l=0}^{n} s(n, l) x^{n} .
$$

Also the generating function for $g_{n}^{k}(n)$ is given by

$$
G(t)=\prod_{i=1}^{n}[n-j(1-t)]
$$

In this article, in Section 2, we derive a generalization of some results given in [1], for the number of paths of order $k, g^{k}\left(a_{1}, \cdots, a_{n} ; m\right)$, when $m \neq n$. The generating function of $g^{k}\left(a_{1}, \cdots, a_{n} ; m\right)$ is given. In Section 3 , we find the probability distribution for $g^{k}\left(a_{1}, \cdots, a_{n} ; m\right)$ and study some of their properties. The moment generating function, skewness and kurtosis for $g^{k}\left(a_{1}, \cdots, a_{n} ; m\right)$ are investigated. Moreover special case and numerical results are given in Section 4.

\section{Main Results}

Theorem 1. The number of paths of order $k$ is given by 


$$
g^{k}\left(a_{1}, a_{2}, \cdots, a_{n} ; m\right)=(-1)^{k} \sum_{i=k}^{n}\left(\begin{array}{l}
i \\
k
\end{array}\right) s_{\bar{a}}(n, n-i) m^{n-i} .
$$

Proof. Using (5) in (8), we get

$$
\begin{aligned}
& g^{k}\left(a_{1}, a_{2}, \cdots, a_{n} ; m\right) \\
= & (-1)^{k} \sum_{i=k}^{n}\left(\begin{array}{l}
i \\
k
\end{array}\right)\left\{s_{\bar{a}}(n-1, n-i-1)-a_{n} s_{\bar{a}}(n-1, n-i)\right\} m^{n-i} \\
= & (-1)^{k} \sum_{i=k}^{n-1}\left(\begin{array}{l}
i \\
k
\end{array}\right) s_{\bar{a}}(n-1, n-i-1) m^{n-i}-(-1)^{k} \sum_{i=k}^{n} a_{n}\left\{\left(\begin{array}{c}
i-1 \\
k-1
\end{array}\right)+\left(\begin{array}{c}
i-1 \\
k
\end{array}\right)\right\} s_{\bar{a}}(n-1, n-i) m^{n-i} \\
= & (-1)^{k} \sum_{i=k}^{n-1}\left(\begin{array}{l}
i \\
k
\end{array}\right) s_{\bar{a}}(n-1, n-i-1) m^{n-i}-\left\{(-1)^{k} a_{n} \sum_{i=k+1}^{n}\left(\begin{array}{c}
i-1 \\
k
\end{array}\right)\right. \\
& \left.\times s_{\bar{a}}(n-1, n-i) m^{n-i}+(-1)^{k} a_{n} \sum_{i=k}^{n}\left(\begin{array}{c}
i-1 \\
k-1
\end{array}\right) s_{\bar{a}}(n-1, n-i) m^{n-i}\right\} \\
= & (-1)^{k} \sum_{i=k}^{n-1}\left(\begin{array}{l}
i \\
k
\end{array}\right) s_{\bar{a}}(n-1, n-i-1) m^{n-i}-(-1)^{k} a_{n} \sum_{i=k}^{n-1}\left(\begin{array}{l}
i \\
k
\end{array}\right) s_{\bar{a}}(n-1, n-i-1) \\
& \times m^{n-i-1}-(-1)^{k} a_{n} \sum_{i=k-1}^{n-1}\left(\begin{array}{c}
i \\
k-1
\end{array}\right) s_{\bar{a}}(n-1, n-i-1) m^{n-i-1} \\
= & \left(m-a_{n}\right) g^{k}\left(a_{1}, \cdots, a_{n-1} ; m\right)+a_{n} g^{k-1}\left(a_{1}, \cdots, a_{n-1} ; m\right) .
\end{aligned}
$$

This by virtue of (1) completes the proof of (8).

Theorem 2. The generating function of the number of paths of order $k$ is given by

$$
G^{(n)}(n ; m)=\prod_{i=1}^{n}\left(m-a_{i}(1-t)\right)
$$

Proof. Let the generating function of the number of paths of order $k$ be denoted by

$$
G^{(n)}(n ; m)=\sum_{k=0}^{n} g^{k}\left(a_{1}, \cdots, a_{n} ; m\right) t^{k}
$$

Using (1), we obtain

$$
\begin{aligned}
G^{(n)}(n ; m) & =\sum_{k=1}^{n} a_{n} g^{k-1}(n-1 ; m) t^{k}+\left(m-a_{n}\right) \sum_{k=0}^{n} g^{k}(n-1 ; m) t^{k}, \\
& =a_{n} t G^{(n-1)}(n-1 ; m)+\left(m-a_{n}\right) G^{(n-1)}(n-2 ; m) \\
& =\left(m-a_{n}+a_{n} t\right) G^{(n-1)}(n-1 ; m),
\end{aligned}
$$

and hence we get

$$
G^{(n)}(n ; m)=\prod_{i=1}^{n}\left(m-a_{i}(1-t)\right) G^{(0)}(0 ; m),
$$

where $G^{(0)}(0 ; m)=g^{0}(0 ; m)=1$. This completes the proof.

From (9), we get

$$
G^{(n)}(n ; m)=a_{1}\left(t-\frac{a_{1}-m}{a_{1}}\right) a_{2}\left(t-\frac{a_{2}-m}{a_{2}}\right) \cdots a_{n}\left(t-\frac{a_{n}-m}{a_{n}}\right)=\prod_{i=1}^{n} a_{i} \sum_{k=0}^{n} s_{\bar{\alpha}}(n, k) t^{k},
$$

where $\alpha_{i}=-\left(\frac{m-a_{i}}{a_{i}}\right), \bar{\alpha}:=\left(\alpha_{1}, \alpha_{2}, \cdots, \alpha_{n}\right)$ and hence we have 


$$
g^{k}\left(a_{1}, \cdots, a_{n} ; m\right)=\prod_{i=1}^{n} a_{i} s_{\bar{\alpha}}(n, k),
$$

where $\alpha_{i}=-\left(\frac{m-a_{i}}{a_{i}}\right), i=1, \cdots, n$.

For the special case $a_{i}=i, m=n$, we get

$$
g_{n}^{k}(n)=n ! s_{\bar{\alpha}}(n, k)
$$

where $\alpha_{i}=-\left(\frac{n-i}{i}\right), i=1, \cdots, n$.

From (6) and (12), we have the identity

$$
(-1)^{k} \sum_{m=k}^{n}\left(\begin{array}{l}
m \\
k
\end{array}\right) s(n+1, n+1-m) n^{n-m}=n ! s_{\bar{\alpha}}(n, k),
$$

where $\alpha_{i}=-\left(\frac{n-i}{i}\right), i=1, \cdots, n$.

\section{Some Applications}

Let $X$, be the number of paths along $A_{n m}$, then by virtue of (8) we have

$$
\begin{aligned}
P(X=k) & =(-1)^{k} \sum_{i=k}^{n}\left(\begin{array}{l}
i \\
k
\end{array}\right) s_{\bar{a}}(n, n-i) \frac{m^{n-i}}{m^{n}} \\
& =(-1)^{k} \sum_{i=k}^{n}\left(\begin{array}{l}
i \\
k
\end{array}\right) \frac{s_{\bar{a}}(n, n-i)}{m^{i}}, k=0,1, \cdots, n .
\end{aligned}
$$

On the other hand the moment generating function of the random variable $X$ denoted by $M_{X}(t)$, is given by the following theorem.

Theorem 3. The moment generating function of $X$, is given by

$$
M_{X}(t)=\sum_{n=0}^{\infty} \sum_{i=0}^{n} \frac{(-1)^{i} i !}{n ! m^{i}} S(n, i) s_{\bar{a}}(n, n-i) t^{n} .
$$

Proof. We begin by the definition of the moment generating function as follows.

$$
\begin{aligned}
M_{X}(t) & =\sum_{k=0}^{n} \mathrm{e}^{k t} f(k)=\sum_{k=0}^{n} \mathrm{e}^{k t}(-1)^{k} \sum_{i=k}^{n}\left(\begin{array}{l}
i \\
k
\end{array}\right) \frac{s_{\bar{a}}(n, n-i)}{m^{i}} \\
& =\sum_{i=0}^{n} \sum_{k=0}^{i}(-1)^{k}\left(\begin{array}{l}
i \\
k
\end{array}\right) \mathrm{e}^{k t} \frac{s_{\bar{a}}(n, n-i)}{m^{i}}=\sum_{i=0}^{n}\left(1-\mathrm{e}^{t}\right)^{i} \frac{S_{\bar{a}}(n, n-i)}{m^{i}} \\
& =\sum_{i=0}^{n} \sum_{n=i} \frac{(-1)^{i} i !}{n ! m^{i}} S(n, i) s_{\bar{a}}(n, n-i) t^{n} \\
& =\sum_{n=0}^{\infty} \sum_{i=0}^{n} \frac{(-1)^{i} i !}{n ! m^{i}} S(n, i) s_{\bar{a}}(n, n-i) t^{n}
\end{aligned}
$$

This completes the proof.

Corollary 1. The jth moments of $X$ is

$$
E\left[X^{j}\right]=\sum_{i=0}^{n} \frac{(-1)^{i} i !}{m^{i}} S(j, i) s_{\bar{a}}(n, n-i) .
$$

Proof. The $j$ th moments can be obtained from the moment generating function, $M_{X}(t)$ where 


$$
E\left[X^{j}\right]=\left[\frac{\mathrm{d}^{n}}{\mathrm{~d} t^{n}} M_{X}(t)\right]_{t=0}
$$

This completes the proof.

Then from (16), we can calculate the mean and variance for the random variable $X$ as follows.

$$
\begin{aligned}
\mu=E(X) & =\sum_{i=0}^{n} \frac{(-1)^{i} i !}{m^{i}} S(1, i) s_{\bar{a}}(n, n-i)=-\frac{s_{\bar{a}}(n, n-1)}{m} . \\
E\left(X^{2}\right) & =\sum_{i=0}^{n} \frac{(-1)^{i} i !}{m^{i}} S(2, i) s_{\bar{a}}(n, n-i) \\
& =-\frac{S(2,1) s_{\bar{a}}(n, n-1)}{m}+\frac{2 ! S(2,2) s_{\bar{a}}(n, n-2)}{m^{2}} \\
& =-\frac{s_{\bar{a}}(n, n-1)}{m}+\frac{2 s_{\bar{a}}(n, n-2)}{m^{2}},
\end{aligned}
$$

hence the variance is given by

$$
\begin{aligned}
\operatorname{Var}(X) & =E\left[X^{2}\right]-\mu^{2}=-\frac{s_{\bar{a}}(n, n-1)}{m}+\frac{2 s_{\bar{a}}(n, n-2)}{m^{2}}-\left(-\frac{s_{\bar{a}}(n, n-1)}{m}\right)^{2} \\
& =-\frac{s_{\bar{a}}(n, n-1)}{m^{2}}\left[s_{\bar{a}}(n, n-1)+m\right]+\frac{2 s_{\bar{a}}(n, n-2)}{m^{2}} .
\end{aligned}
$$

Corollary 2. The Skewness and kurtosis for the random variable $X$ are given by

$$
\text { Skewness }=\frac{\mu_{3}}{\mu_{2}^{3 / 2}}, \text { kurtosis }=\frac{\mu_{4}}{\mu_{2}^{2}}
$$

where

$$
\begin{gathered}
\mu_{2}=-\frac{s_{\bar{a}}(n, n-1)}{m}+\frac{2 s_{\bar{a}}(n, n-2)}{m^{2}}-\frac{s_{\bar{a}}^{2}(n, n-1)}{m^{2}}, \\
\mu_{3}=-\frac{s_{\bar{a}}(n, n-1)}{m}-\frac{3 s_{\bar{a}}^{2}(n, n-1)}{m^{2}}-\frac{2 s_{\bar{a}}^{3}(n, n-1)}{m^{3}}+\frac{6 s_{\bar{a}}(n, n-2)}{m^{2}} \\
+\frac{6 s_{\bar{a}}(n, n-2) s_{\bar{a}}(n, n-1)}{m^{3}}-\frac{6 s_{\bar{a}}(n, n-3)}{m^{3}}, \\
\mu_{4}=-\frac{s_{\bar{a}}(n, n-1)}{m}-\frac{4 s_{\bar{a}}^{2}(n, n-1)}{m^{2}}-\frac{6 s_{\bar{a}}^{3}(n, n-1)}{m^{3}}-\frac{3 s_{\bar{a}}^{4}(n, n-1)}{m^{4}}+\frac{14 s_{\bar{a}}(n, n-2)}{m^{2}} \\
+\frac{24 s_{\bar{a}}(n, n-2) s_{\bar{a}}(n, n-1)}{m^{3}}+\frac{12 s_{\bar{a}}(n, n-2) s_{\bar{a}}^{2}(n, n-1)}{m^{4}}-\frac{36 s_{\bar{a}}(n, n-3)}{m^{3}} \\
-\frac{24 s_{\bar{a}}(n, n-3) s_{\bar{a}}(n, n-1)}{m^{4}}+\frac{24 s_{\bar{a}}(n, n-4)}{m^{4}} .
\end{gathered}
$$

Proof. We can find the jth moments about the mean by using

$$
\mu_{j}=E\left[(X-E[X])^{j}\right]=\sum_{k=0}^{j}\left(\begin{array}{l}
j \\
k
\end{array}\right)(-1)^{k}(E[X])^{k} E\left[X^{j-k}\right] .
$$

From (16) and (21), we can find the moments $\mu_{2}, \mu_{3}, \mu_{4}$ about mean which can be used to calculate the skweness and kurtosis.

\section{Special Case:}

If $a_{i}=i, m=n$, from (14), we have 


$$
P(X=k)=(-1)^{k} \sum_{i=k}^{n}\left(\begin{array}{l}
i \\
k
\end{array}\right) \frac{s(n+1, n+1-i)}{n^{i}},
$$

and from (16) the jth moments has the form

$$
E\left[X^{j}\right]=\sum_{i=0}^{n} \frac{(-1)^{i} i ! S(j, i) s(n+1, n+1-i)}{n^{i}},
$$

and the mean is given by

$$
\begin{gathered}
\mu=-\frac{s(n+1, n)}{n}=\frac{n+1}{2} . \\
E\left[X^{2}\right]=-\frac{s(n+1, n)}{n}+\frac{2 s(n+1, n-1)}{n^{2}},
\end{gathered}
$$

the variance can be obtained as follows.

$$
\begin{aligned}
\operatorname{Var}(X) & =E\left[X^{2}\right]-\mu^{2}=-\frac{s(n+1, n)}{n}+\frac{2 s(n+1, n-1)}{n^{2}}-\left(-\frac{s(n+1, n)}{n}\right)^{2} \\
& =-\frac{s(n+1, n)}{n^{2}}[s(n+1, n)+n]+\frac{2 s(n+1, n-1)}{n^{2}} \\
& =\frac{(n-1)(n+1)}{6 n} .
\end{aligned}
$$

where we used $s(n, n-1)=-\left(\begin{array}{l}n \\ 2\end{array}\right), s(n, n-2)=\frac{1}{4}(3 n-1)\left(\begin{array}{l}n \\ 3\end{array}\right)$, see [3].

\section{Numerical Results}

Setting $n=5, m=6, \alpha_{i}=i, i=1,2, \cdots, n$. Therefore the numerical values of $s_{\bar{\alpha}}(n, k)$, are reduced to $s(n, k)$, see [4] [5].

From Equation (14), we can find the probability distribution of the number of paths $X$ along $A_{5 \times 6}$ as follows

\begin{tabular}{cccccccc}
\hline$X$ & 0 & 1 & 2 & 3 & 4 & 5 & Total \\
\hline$p(x)$ & 0.09259 & 0.34259 & 0.38889 & 0.15741 & 0.01852 & 0 & 1 \\
\hline
\end{tabular}

From (16), we can compute the 4th moments as follows.

\begin{tabular}{ccccc}
\hline$j$ & 1 & 2 & 3 & 4 \\
\hline$E\left[X^{j}\right]$ & 1.66667 & 3.61111 & 8.88889 & 24.0556 \\
\hline
\end{tabular}

The $4^{\text {th }}$ moments about mean can be obtained as

\begin{tabular}{ccccc}
\hline$j$ & 1 & 2 & 3 & 4 \\
\hline$\mu_{j}$ & 0 & 0.83333 & 0.09259 & 1.83333 \\
\hline
\end{tabular}

The values of mean and variance can be obtained from (17) and (19) as follows.

$$
\mu=1.66667, \operatorname{Var}(X)=0.83333 \text {. }
$$

The skewness and kurtosis, respectively can be obtained from (20) as follows. 


$$
\text { skewness }=0.12172 \text {, } \text { kurtosis }=2.64 \text {. }
$$

\section{References}

[1] Khidr, A.M. and El-Desouky, B.S. (1984) A Symmetric Sum Involving the Stirling Numbers of the First Kind. European Journal of Combinatorics, 5, 51-54. http://dx.doi.org/10.1016/S0195-6698(84)80018-9

[2] Comtet, L. (1972) Nombres de Stirling generaux et fonctions symetriques. Comptes Rendus de l'Académie des Sciences Paris (Series A), 275, 747-750.

[3] Comtet, L. (1974) Advanced Combinatorics: The Art of Finite and Infinite Expansions. D. Reidel Publishing Company, Dordrecht, Holand.

[4] El-Desouky, B.S. (1994) Multiparameter Non-Central Stirling Numbers. The Fibonacci Quarterly, 32, $218-225$.

[5] El-Desouky, B.S. and Cakić, N.P. (2011) Generalized Higher Order Stirling Numbers. Mathematical and Computer Modelling, 54, 2848-2857. http://dx.doi.org/10.1016/j.mcm.2011.07.005

[6] Cakić, N.P., El-Desouky, B.S. and Milovanović, G.V. (2013) Explicit Formulas and Combinatorial Identities for Generalized Stirling Numbers. Mediterranean Journal of Mathematics, 10, 57-72. http://dx.doi.org/10.1007/s00009-011-0169-x 